\section{SAT0112 GUIDELINE-BASED CARE IMPROVES OUTCOMES THAT MATTER TO PATIENTS: TIGHTER CONTROL, LESS SUFFERING, AND GREATER WELL-BEING OVER THE PAST DECADE IN CANADIAN RA PATIENTS}

S. J. Bartlett ${ }^{1,2}$, O. Schieir ${ }^{3}$, M.-F. Valois ${ }^{4}$, B. Haraoui ${ }^{5}$, G. Boire ${ }^{6}$, C. Thorne ${ }^{7}$, C. Hitchon ${ }^{8}$, D. Tin ${ }^{7}$, E. C. Keystone ${ }^{3,9}$, J. Pope ${ }^{10}$, V. P. Bykerk ${ }^{3,11}$ on behalfof CATCH: Canadian Early Arthritis Cohort. ${ }^{1}$ Medicine/ClinEpi \& Rheumatology, McGill University, Montreal, QC, Canada; ${ }^{2}$ Medicine/Rheumatology, Johns Hopkins, Baltimore, United States; ${ }^{3}$ University of Toronto, Toronto, ON; ${ }^{4}$ Clinical Epidemiology, McGill University; ${ }^{5}$ Institute de Rheumatologie, Montreal, QC; ${ }^{6}$ Rheumatologie, Universite de Sherbrooke, Sherbrooke, QC; ${ }^{7}$ Southlake Regional Health Centre, Newmarket, ON; ${ }^{8}$ Rheumatology, University of Manitoba, Winnipeg, $\mathrm{MB} ;{ }^{9}$ Sinai Health System, Toronto, ON; ${ }^{10}$ Western University, London, ON, Canada; ${ }^{11}$ Hospital for Special Surgery, New York, NY, United States

Background: Best practice recommendations can increase the quality of care and improve clinical outcomes, however, the impact of guideline-based care on outcomes that matter most to early RA patients before and after implementation of treat to target (T2T) recommendations has not been evaluated.

Objectives: We compared changes over the first year of treatment in outcomes valued most by RA patients: 1 ) in relation to disease activity; and 2) prior (200710) and subsequent (2011-16) to the release of T2T and Canadian RA Recommendations.

Methods: Data included early RA adults enrolled in CATCH (Canadian Early ArThritis CoHort) between 2007-16 who met 1987/2010 RA criteria with active disease at enrolment. Treatment was at the discretion of the rheumatologist and cohort investigators met annually to discuss ways to improve outcomes. We compared changes in DAS28, pain (0-10), fatigue (0-10), patient global (0-10), and $\mathrm{HAQ}-\mathrm{DI}$ at 6 and 12 months prior to and after the release of guidelines using Cochran-Armitage trend tests and regression.

Results: The sample included 1942 adults who were mostly female (72\%) with a mean (SD) age of 55 (15), 2 (2) comorbidities, and symptom duration of 6 (3) months. At enrollment, almost all (95\%) were in DAS28 moderate disease activity [MDA; $42 \%$ ] or high disease activity [HDA; $53 \%$ ], and were initially treated with csDMARDS (92\%) and MTX (75\%). CDAI, DAS28 and PROs by DAS28 disease levels are shown in the Table.

\begin{tabular}{lllll}
\hline Participant characteristics. & & & & \\
\hline Mean (SD) & Total & LDA & MDA & HDA \\
\hline N (\%) & 1942 & $93(5 \%)$ & $828(42 \%)$ & $1021(53 \%)$ \\
DAS28 & $5.3(1.3)$ & $2.9(0.2)$ & $4.2(0.5)$ & $6.2(0.8)$ \\
CDAI & $28.1(13.8)$ & $10.8(5.3)$ & $19.4(8.0)$ & $36.9(11.8)$ \\
Patient Global (0-10) & $6.0(2.8)$ & $3.2(2.2)$ & $4.9(2.7)$ & $7.2(2.4)$ \\
Pain (0-10) & $5.7(2.8)$ & $3.3(2.3)$ & $4.6(2.6)$ & $6.8(2.4)$ \\
Fatigue (0-10) & $5.4(3.0)$ & $3.8(2.8)$ & $4.6(2.9)$ & $6.1(2.9)$ \\
HAQ-DI (0-3) & $1.1(0.7)$ & $0.6(0.5)$ & $0.8(0.6)$ & $1.4(0.7)$ \\
\hline
\end{tabular}

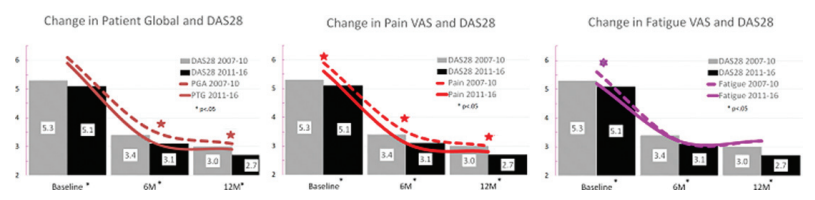

As mean DAS28 decreased over the year, similar improvements in patient global, pain, $\mathrm{HAQ}$, and fatigue were also evident $\left(-3.0,-2.8,-2.3,-0.6\right.$; $\left.\mathrm{p}^{\prime} \mathrm{s}<0.001\right)$. When comparing change in PROs in the two time periods, there were more rapid improvements in patient global and pain at 6 and 12 months (p's $<0.001$; figure 1 ) and similar improvements in $\mathrm{HAQ}$ and fatigue.

Conclusions: Results from this large country-wide study suggest that T2T results in better disease control in the first year of RA with similar improvements in pain, fatigue, and disability-symptoms that patients identify as important-resulting in greater overall well-being. These data offer additional evidence supporting the importance of early identification and rapid control of RA to improve long-term outcomes and QOL.

Acknowledgements: Sponsors: Amgen \& Pfizer-Founding sponsors 2007+; UCB, AbbVie 2011+; Medexus 2013+; Eli Lilly, Sanofi- Genzyme 2016+; Merck 2017+; BMS 2011-2014, Hoffmann-LaRoche, Janssen 2011-2016

Disclosure of Interest: None declared

DOI: 10.1136/annrheumdis-2018-eular.5579

\section{SAT0113 \\ CLUSTERIN SERUM LEVELS ARE ELEVATED IN PATIENTS WITH EARLY RHEUMATOID ARTHRITIS AND PREDICT DISEASE ACTIVITY AND TREATMENT RESPONSE}

T. Lennerová ${ }^{1,2}$, H. Mann ${ }^{1,2}$, O. Rủžičková ${ }^{1,2}$, O. Šléglová ${ }^{1,2}$, L. Vernerová ${ }^{1}$ M. Tomčík ${ }^{1,2}, K_{\text {. Pavelka }}{ }^{1,2}$, J. Vencovskýy ${ }^{1,2}$, L. Šenolt ${ }^{1,2}$. Institute of Rheumatology, Prague, Czech Republic; ${ }^{2}$ Department of Rheumatology, 1 st Faculty of Medicine, CharlesUniversity, Prague, Czech Republic

Background: Clusterin (also known as apolipoprotein $\mathrm{J}$ ) is an extracellular chaperone that participates in a number of biological processes, including inflammation and apoptosis. Recent data suggest its possible protective role in the development of bone erosions and autoimmune disorders.

Objectives: The aim of our study was to analyze the serum levels of clusterin in patients with early rheumatoid arthritis (RA) and in healthy individuals, and to examine their potential association with disease activity and treatment response. Methods: The serum levels of clusterin were determined by ELISA (BioVendor) in 56 patients with early RA before and three months after initiation of treatment, and in 56 age /sex-matched healthy subjects. Disease activity was evaluated by Clinical Disease Activity Index (CDAI), Simplified Disease Activity Index (SDAI) and 28-joint Disease Activity Score (DAS28). Response to therapy was assessed based on the SDAI/CDAI definition. Receiver operating characteristic (ROC) curve analysis of clusterin levels was performed to predict disease activity and treatment response after six months of treatment. The area under the curve (AUC) and the $95 \%$ confidence interval $(\mathrm{Cl})$ were calculated. Data are presented as mean $\pm S D$.

Results: Clusterin levels at baseline were significantly higher in patients with early RA than in healthy individuals $(75.1 \pm 12.4$ vs $56.7 \pm 9.7, p<0.001)$. After three months of therapy, the concentrations of clusterin decreased and reached levels comparable to those in healthy subjects $(57.7 \pm 9.7$ vs $56.7 \pm 9.7, p>0.05)$. Although there was no association between clusterin levels and disease activity at baseline clusterin levels positively correlated with SDAl and CDAI at month $3(r=0.269$, $p=0.047$ and $r=0.294, p=0.030$, respectively $)$ and at month $6(r=0.339, p=0.013$ and $r=0.318, p=0.021$, respectively) after treatment initiation. Using ROC analysis, clusterin baseline levels predicted remission and low disease activity according to SDAI (AUC $=0.709(95 \% \mathrm{Cl} 0.548 ; 0.869), \mathrm{p}=0.019)$ and CDAI (AUC = $0.829(95 \% \mathrm{Cl} 0.721 ; 0.937), \mathrm{p}<0.001)$, and major treatment response after 6 months of therapy (AUC $=0.696$ ( $95 \% \mathrm{Cl} 0.549 ; 0.842), \mathrm{p}=0.015$ for both).

Conclusions: We demonstrate elevated serum concentrations of clusterin in patients with early rheumatoid arthritis and suggest clusterin as a biomarker for predicting disease activity and treatment response.

Acknowledgements: Supported by the project of MHCR for conceptual development of research organization 00023728, research project SVV 260373 and project GAUK No. 534217.

Disclosure of Interest: None declared

DOI: 10.1136/annrheumdis-2018-eular.3626

\section{SAT0114 TRENDS IN THE INCIDENCE OF SOLID TUMORS IN PATIENTS WITH RHEUMATOID ARTHRITIS IN SPAIN. A NATIONAL OBSERVATIONAL COHORT STUDY.}

V. Villaverde ${ }^{1}$, M. Fernandez-Prada ${ }^{2}$, R. Mazzucchelli ${ }^{3}$, E. Perez-Fernandez ${ }^{3}$, C. Macía-Villa ${ }^{4}$, M. Espinosa ${ }^{5}$, J. Quiros ${ }^{3}$, M. Peña ${ }^{3}$, J. L. Morell ${ }^{6}$, C. MoradoQuiñoa $^{7}$, C. Martínez-Prada ${ }^{7}$, C. Barbadillo ${ }^{5}$, H. Godoy ${ }^{5}$, N. Crespi ${ }^{8}$, O. GuzónIllescas ${ }^{3}$, M. Galindo ${ }^{9}$, A. Herranz ${ }^{10}$, A. García-Vadillo ${ }^{11} .{ }^{1}$ H.U. Móstoles, Madrid; ${ }^{2}$ H. U. de Guadalajara, Guadalajara; ${ }^{3}$ H.U.Fundación Alcorcón; ${ }^{4}$ H.U.Severo Ochoa; ${ }^{5}$ H.U. Puerta de Hierro; ${ }^{6}$ H.U.Ramón y Cajal; ${ }^{7}$ H.U. Clínico San Carlos; ${ }^{8} \mathrm{C}$. S. La Rivota; ${ }^{9}$ H.U. 12 de Octubre; ${ }^{10}$ H.U. del Henares; ${ }^{11}$ H.U.La Princesa, Madrid, Spain

Background: During the last 20 years, the rheumatoid arthritis (RA) treatment has changed. Considering the increasing use of biological immunomodulators to treat this desease and the concern that immunomodulation may alter cancer risk, it's important to know the tumors incidence in RA.

Objectives: To analyze the incidence and trend of hospital admissions for solid tumors in patients with RA in Spain during the period between 1999 and 2015

Methods: This is a retrospective population based study. We analized a national administrative database that includes a Minimum Basic Data Set (MBDS) of all hospital admissions of RA patients. Period: 1999 to 2015. We selected the MBDS for solid tumors. Cases were identified by the presence in primary and secondary diagnosis of ICD 9 codes. The population at risk was estimated through the 